\title{
Applying combinatorial results to products of conjugacy classes
}

\author{
Rachel Deborah Camina \\ Fitzwilliam College, Cambridge, CB3 ODG, UK \\ rdc26@cam.ac.uk
}

\begin{abstract}
Let $K=x^{G}$ be the conjugacy class of an element $x$ of a group $G$ and suppose $K$ is finite. We study the increasing sequence of natural numbers $\left\{\left|K^{n}\right|\right\}_{n \geq 1}$ and consider restrictions on this sequence and the algebraic consequences. In particular, we prove that if $\left|K^{2}\right|<\frac{3}{2}|K|$ or if $\left|K^{4}\right|<2|K|$ then $K^{n}$ is a coset of the normal subgroup $[x, G]$ for all $n \geq 2$ or 4 , respectively. We then use these results to contribute to conjectures about the solubility of $\langle K\rangle$ when $K^{n}$ satisfies certain conditions.
\end{abstract}

\section{Introduction}

Suppose $K=x^{G}$ is the conjugacy class of an element $x$ in a group $G$ and suppose $K$ is finite. Let $n$ be a natural number. The study of $K^{n}=$ $\left\{x_{1} \cdots x_{n} \mid x_{i} \in K\right\}$, the powers of the class $K$, has long interested mathematicians, see for example [1]. More recently Guralnick and Navarro [7] considered the case when $G$ is finite and $K^{2}$ is a conjugacy class, this led to the situation when $K^{n}$ is a conjugacy class, and $n$ is an integer at least 2, being studied in [3]. Note, assuming $G$ finite and $K^{n}$ a conjugacy class, then $K^{n}$ is equal to $\left(x^{n}\right)^{G}$ and since $\left|K^{n}\right| \geq|K|$ and $C_{G}(x) \leq C_{G}\left(x^{n}\right)$ (where $C_{G}(x)$ denotes the centraliser of $x$ in $\left.G\right)$ it follows that $\left|K^{n}\right|=|K|$. In this article instead of considering the sets $K^{n}$ we consider the increasing sequence of natural numbers $\left\{\left|K^{n}\right|\right\}_{n \geq 1}$ and see how restrictions on this sequence lead to algebraic consequences. For example if the sequence stops, that is $\left|K^{m}\right|=\left|K^{m+1}\right|$ for some $m \geq 1$, then $K^{r}=x^{r} N$ for all $r \geq m$ where 
$N$ is the normal subgroup $[x, G]=\langle[x, g] \mid g \in G\rangle$. In particular if $\left|K^{2}\right|=|K|$ then $K=x N$. Applying the 'Freiman Inverse Problem for $\kappa<\frac{3}{2}$ ', allows us to generalise these ideas as the following theorem shows.

Theorem A. Suppose $K=x^{G}$ is a conjugacy class of an element $x$ of a group $G$ and $K$ is finite. Let $N$ denote the normal subgroup $[x, G]$.

(i) If $\left|K^{2}\right|=\mu|K|$ with $\mu<\frac{3}{2}$ then $K^{r}=x^{r} N$ for all $r \geq 2$.

(ii) If $\left|K^{3}\right|=\frac{3}{2}|K|$ then $K^{r}=x^{r} N$ for all $r \geq 2$.

(iii) If $\left|K^{4}\right|=\mu|K|$ with $\mu<2$ then $K^{r}=x^{r} N$ for all $r \geq 4$.

(iv) If $\left|K^{5}\right|=2|K|$ then $K^{r}=x^{r} N$ for all $r \geq 4$.

For example if $A_{4}$ is the alternating group of degree 4 and $K$ is the conjugacy class of the double transposition $x=(1,2)(3,4)$, then $\left|K^{2}\right|=4=$ $\frac{4}{3}|K|$ and indeed $K^{2}=K K^{-1}=\left[x, A_{4}\right]$ is a normal subgroup of $A_{4}$. An example for (ii) is given by the conjugacy class $K$ of the 3 -cycle $x=(1,2,3)$ in $S_{3}$ the symmetric group of degree 3 . In this case $K^{2}=K K^{-1}=\left[x, S_{3}\right]$ is the normal subgroup of $S_{3}$ and $\left|K^{3}\right|=\left|K^{2}\right|=\frac{3}{2}|K|$. Note (i) is best possible in the following sense. Let $D_{2 n}$ be the dihedral group of order $2 n$ with rotation $r$ of order $n$ and suppose $n \geq 5$. Let $K$ be the conjugacy class of $r$ in $D_{2 n}$, then $\left|K^{2}\right|=3=\frac{3}{2}|K|$ but $\left|K^{r}\right|>\left|K^{2}\right|$ for all $r \geq 3$. Consideration of the conjugacy class of a rotation of order 8 in $D_{16}$ illustrates (iv).

In [3] the authors propose the following conjecture.

Conjecture. [3, Conjecture 2] Let $G$ be a finite group and $K$ a conjugacy class. If $K^{n}=D \cup D^{-1}$ for some natural number $n \geq 2$ and $D$ a conjugacy class, then $\langle K\rangle$ is soluble.

The authors prove that in this scenario $|D|=|K| / 2$ or $|D|=|K|$. For the cases when $D=D^{-1}$ or $|D|=|K| / 2$ they prove that $\langle K\rangle$ is soluble. They also show that if $K^{2}=K \cup K^{-1}$ then $\langle K\rangle$ is soluble and $x$ is a $p$-element for some prime $p$. More recently Beltrán and Felipe [4, Theorem B] have shown that if $K^{n}=K \cup K^{-1}$ then $\langle K\rangle$ is soluble. Examples of when these situations arise are given in [3, Example 3] and [4, Example 3.2]. By applying Theorem $\mathrm{A}$ and $[4$, Theorem $\mathrm{A}]$ we prove the following, giving more evidence for the conjecture above.

Theorem B. Suppose $G$ is a finite group and $K$ a conjugacy class such that $K^{n}=D \cup D^{-1}$ for some conjugacy class $D$ and $n \geq 4$, then $\langle K\rangle$ is soluble.

We also show that in the situation above $D^{3} \subseteq D \cup D^{-1}$. 
In [5] the authors consider the product $K K^{-1}$ for $K$ a conjugacy class of a finite group $G$. In particular they study when $K K^{-1}=\{1\} \cup D \cup D^{-1}$ and when $K K^{-1}=\{1\} \cup D$ for $D$ a conjugacy class. They prove that in these cases $G$ is not a nonabelian simple group [5, Theorem A] and they conjecture that $\langle K\rangle$ is soluble. Applying a combinatorial result of Tao [8], which considers when $X X^{-1}$ is a group given that $X$ is a finite subset of a nonabelian group, gives the following which adds to this line of research.

Theorem C. Suppose $K=x^{G}$ is a conjugacy class of an element $x$ of a finite group $G$ and

(i) $K K^{-1}=\{1\} \cup D$ for a conjugacy class $D$ and $|D|+1<\frac{3}{2}|K|$ or

(ii) $K K^{-1}=\{1\} \cup D \cup D^{-1}$ for a conjugacy class $D$ and $2|D|+1<\frac{3}{2}|K|$.

Then $\langle D\rangle$ is p-elementary abelian for a prime $p$ and $\langle K\rangle=\langle x\rangle\langle D\rangle$ is metabelian.

\section{The sequence $\left\{\left|K^{n}\right|\right\}_{n \geq 1}$.}

Recall, the kernel of a finite subset $S$ of a group $G$ is defined to be

$$
\operatorname{ker}(S)=\{x \in G: x S=S\} .
$$

Clearly $\operatorname{ker}(S)$ is a subgroup of $G$ and as $S$ is a union of cosets of $\operatorname{ker}(S)$ its order divides $|S|$. If $S$ is a normal subset then $\operatorname{ker}(S)$ is a normal subgroup. Furthermore, a recent result $[7$, Theorem $\mathrm{B}(\mathrm{a})]$ gives that if $S$ is a conjugacy class then $\operatorname{ker}(S)$ is soluble. The notion of a kernel has appeared in the literature many times, under various different names.

The following lemma is a first illustration of how a restriction on the sequence $\left\{\left|K^{n}\right|\right\}_{n \geq 1}$ determines structural properties of $K$. The ideas are known but are presented here for completeness.

Lemma 1. Suppose $K=x^{G}$ is a conjugacy class of a group $G$ and $K$ is finite. Suppose $\left|K^{n}\right|=\left|K^{m}\right|$ for some $n<m$. Then $K^{r}=x^{r} N$ for all $r \geq n$ where $N=[x, G]$ is a normal subgroup of $G$. If $\left|K^{n}\right|=\left|K^{m}\right|<|G|$ then $N$ is a proper normal subgroup.

Proof. As $\left|K^{n}\right|=\left|K^{m}\right|$ it follows that $\left|K^{n}\right|=\left|K^{n+1}\right|$. So, $x K^{n}=K^{n+1}=$ $x^{g} K^{n}$ for all $g \in G$ and thus $N=\langle[x, g]\rangle=[x, G] \leq \operatorname{ker}\left(K^{n}\right)$ and $|N|$ divides $\left|K^{n}\right|$. In particular, when $\left|K^{n}\right|<|G|$ it follows that $N$ is proper. Note $K \subseteq x N$ so $K^{n} \subseteq x^{n} N$. But $|N| \leq\left|K^{n}\right| \leq|N|$, so $K^{n}=x^{n} N$. As $\left|K^{r}\right| \geq\left|K^{n}\right|$ for all $r \geq n$ but also $K^{r} \subseteq x^{r} N$ it follows that $K^{r}=x^{r} N$ as required. 
The following useful result is due to Freiman. Although originally proved in 1973, [6] gives a more recent exposition.

Theorem 1. (Freiman inverse problem for $\kappa<\frac{3}{2}$ ) [6] Suppose $A$ is a finite subset of a nonabelian group $G$ and $\left|A^{2}\right|<\frac{3}{2}|A|$, then the following hold.

(a) The set $H=A^{-1} A=A A^{-1}$ is an $A$-invariant subgroup of $G$ and $A^{2}$ is a coset of $H$.

(b) If $A^{2} \cap H \neq \emptyset$ then $A^{2}=H$.

We apply this to conjugacy classes. Note if $K=x^{G}$ is a conjugacy class then $K K^{-1}=K^{-1} K$. This is because if $a b \in K K^{-1}$ with $a \in K$ and $b \in K^{-1}$ then $b a \in K^{-1} K$ and as $K^{-1} K$ is normal $a b=(b a)^{a^{-1}} \in K^{-1} K$. Further if $K K^{-1}$ is a subgroup then $K K^{-1}=[x, G]$. Also if $G$ is abelian the following result is trivially true.

Proposition 1. Suppose $K=x^{G}$ is a finite conjugacy class of a group $G$ and $\left|K^{2}\right|=\mu|K|$ with $\mu<\frac{3}{2}$. Then $N=K K^{-1}=[x, G]$ is a normal subgroup of $G$ with $|N|=\mu|K|$ and $K^{r}=x^{r} N$ for all $r \geq 2$.

Proof. By the previous theorem $N=K K^{-1}$ is a subgroup, so $N=[x, G]$, and $|N|=\left|K^{2}\right|$. As $K \subseteq x N$ it follows that $K^{2} \subseteq x^{2} N$ and, by orders, $K^{2}=x^{2} N$. As $\left|K^{r}\right| \geq\left|K^{2}\right|$ for all $r \geq 2$ but also $K^{r} \subseteq x^{r} N$ we have $K^{r}=x^{r} N$ for all $r \geq 2$.

Motivated by the situation described in the introduction, that is when $K^{n}=D \cup D^{-1}$ where both $K$ and $D$ are conjugacy classes and $|D|=|K|$, we now investigate the case when $\left|K^{n}\right|=2|K|$. First a lemma which is adapted from [2, page 29].

Lemma 2. Let $K$ be a finite conjugacy class of a group $G$ and suppose $\left|K K^{-1} K\right|<2|K|$. Then $H=K K^{-1}$ is a normal subgroup of $G$.

Proof. Clearly $1 \in K K^{-1}$. Suppose $h, g \in H$ then as $|H K|<2|K|$ it follows that $h K \cap g K \neq \emptyset$ and so there exists $a, b \in K$ such that $h a=g b$. Thus $g^{-1} h=b a^{-1} \in K K^{-1}=H$, it follows that $H$ is a subgroup.

We apply this lemma to our situation.

Proposition 2. Suppose $K=x^{G}$ is a finite conjugacy class of a group $G$.

(i) If $\left|K^{4}\right|=\mu|K|$ for some $\mu<2$ then $N=K K^{-1}$ is a normal subgroup of $G$ with $|N|=\mu|K|$ and $K^{r}=x^{r} N$ for all $r \geq 4$.

(ii) If $\left|K^{m}\right|=2|K|$ for some $m \geq 5$ then $N=[x, G]$ is a normal subgroup of $G$ with $N=2|K|$ and $K^{r}=x^{r} N$ for all $r \geq 4$. 
Proof. (i) First note that if $G$ is abelian then $\mu=1$ and the result trivially holds. So assume $G$ not abelian. Now $\left|K^{2}\right| \leq\left|K^{4}\right|=\mu|K|$, so $\left|K^{2}\right|=\lambda|K|$ for some $\lambda \leq \mu$. If $\lambda<\frac{3}{2}$ then the result follows from Proposition 1. So we assume $\frac{3}{2}|K| \leq\left|K^{2}\right|<2|K|$.

Now $\left|K^{4}\right|=\nu\left|K^{2}\right|$ for some $\nu$ and as $\left|K^{2}\right| \geq \frac{3}{2}|K|$ it follows that $2|K|>$ $\nu\left|K^{2}\right| \geq \frac{3 \nu}{2}|K|$ and so $\nu<\frac{4}{3}<\frac{3}{2}$. Thus $H=K^{2} K^{-2}$ is a finite group of order $\nu\left|K^{2}\right|$ by Theorem 1 . Note that $K K^{-1}=K^{-1} K$ and so $H=K K^{-1} K K^{-1}$ and $\left|K K^{-1} K\right| \leq|H|<2|K|$. So, $K K^{-1}=N$ is a group by Lemma 2 . Moreover $N=H$. As $K \subseteq x N$ for $x \in K$ this forces $K^{n} \subseteq x^{n} N$ for all $n$, and by orders, $K^{r}=x^{r} N$ for all $r \geq 4$.

(ii) If $\left|K^{4}\right|<2|K|$ then by (i) it follows that $\left|K^{n}\right|<2|K|$ for all $n \geq 4$. Thus $\left|K^{4}\right|=2|K|$ and the result follows from Lemma 1 .

Proof of Theorem A. (i) follows from Proposition 1. Suppose $\left|K^{3}\right|=$ $\frac{3}{2}|K|$. If $\left|K^{2}\right|<\left|K^{3}\right|$, then applying (i) gives that $\left|K^{3}\right|=\left|K^{2}\right|<\frac{3}{2}|K|$, a contradiction. So $\left|K^{2}\right|=\left|K^{3}\right|$ and applying Lemma 1 gives (ii). Finally, (iii) and (iv) follow from Proposition 2(i) and (ii) respectively.

We return to considering $K^{n}=D \cup D^{-1}$ with $K$ and $D$ conjugacy classes, $D \neq D^{-1}$ and $|K|=|D|$. We have two cases (i) when $\left|K^{n-1}\right|<2|K|$ and (ii) when $\left|K^{m}\right|=2|K|$ for some $m<n$. The previous proposition says that if $n>4$ then we are in the second case. The next lemma says this also holds if $n=4$.

Lemma 3. Suppose $K$ is a conjugacy class of a finite group $G$ and $K^{4}=D \cup$ $D^{-1}$ with $D$ a conjugacy class, $D \neq D^{-1}$ and $|K|=|D|$. Then $\left|K^{2}\right|=2|K|$.

Proof. Note $|K|<\left|K^{2}\right|$ by Lemma 1. It follows that $K^{2}$ is not a single conjugacy class, since if it were $K^{2}=\left(x^{2}\right)^{G}$ but then $\left|K^{2}\right| \leq|K|$ as $C_{G}(x) \leq$ $C_{G}\left(x^{2}\right)$. Suppose $|K|<\left|K^{2}\right|<2|K|$. As $K^{2}$ is not a single conjugacy class there exists $y \in K^{2}$ with $\left|y^{G}\right|<|K|$, thus $\left|C_{G}(y)\right|>\left|C_{G}(x)\right|$. Now, $y^{2} \in K^{4}$ so $\left|\left(y^{2}\right)^{G}\right|=|K|$. This implies $\left|C_{G}\left(y^{2}\right)\right|=\left|C_{G}(x)\right|<\left|C_{G}(y)\right|$ which contradicts $C_{G}(y) \leq C_{G}\left(y^{2}\right)$.

Note the above proof also shows that in this case $K^{2}$ is a union of two conjugacy classes of size $|K|$.

We have the following theorem.

Theorem 2. Let $K=x^{G}$ be a conjugacy class of a finite nontrivial group $G$, let $N$ denote the normal subgroup $[x, G]$ and let $n \geq 4$ be an integer. Suppose $K^{n}=D \cup D^{-1}$ with $D$ a conjugacy class, $D \neq D^{-1}$ and $|D|=|K|$. 
(i) If $n>4$, then $K^{r}=x^{r} N$ for all $r \geq 4$ and $N$ is proper.

(ii) If $n=4$, then $K^{r}=x^{r} N$ for all $r \geq 2$ and $N$ is proper.

Proof. Note that $K^{n}=D \cup D^{-1} \neq G$. So (i) follows from Proposition 2(ii). For (ii), applying Lemma 3 gives that $\left|K^{2}\right|=2|K|=\left|K^{4}\right|$ and the result follows from Lemma 1 .

This yields Theorem B which confirms [3, Conjecture 2] for $n \geq 4$.

Proof of Theorem B. If $D=D^{-1}$ the result follows from [3, Theorem A]. By [3, Theorem C] either $|D|=|K| / 2$ or $|D|=|K|$ and in the first case the result follows. So we assume $D \neq D^{-1}$ and $|D|=|K|$. By the previous theorem $K^{n}=x^{n} N=D \cup D^{-1}$ where $N=[x, G]$. Applying [4, Theorem A] gives that $N$ is soluble. As $\langle K\rangle=\langle x\rangle N$ it follows that $\langle K\rangle$ is also soluble. $\square$

We also have the following structural information.

Proposition 3. Suppose $K$ and $D$ are conjugacy classes of a finite group satisfying $K^{n}=D \cup D^{-1}$ with $|D|=|K|$ and $n \geq 4$. Then $D^{3} \subseteq D \cup D^{-1}$.

Proof. If $D=D^{-1}$ then $D^{3}=D$ by [3, Theorem 4], so assume $D \neq D^{-1}$. As $n \geq 4$ by Theorem 2 it follows that $K^{n}=x^{n} N=D \cup D^{-1}=x^{-n} N$, where $N=[x, G]$. Thus

$$
K^{2 n}=x^{2 n} N=N=\left(D \cup D^{-1}\right)\left(D \cup D^{-1}\right)=D^{2} \cup D^{-1} D \cup D^{-2},
$$

as $D D^{-1}=D^{-1} D$. Without loss of generality we assume $D=\left(x^{n}\right)^{G}$. As $D^{-1} D \subseteq N$ it follows that $D N=x^{n} N$. Thus $D^{3} \subseteq D N=x^{n} N=D \cup D^{-1}$

\section{The normal subset $K K^{-1}$.}

In [5] the authors consider the product $K K^{-1}$ for $K$ a conjugacy class. In particular they study when $K K^{-1}=\{1\} \cup D \cup D^{-1}$ and when $K K^{-1}=$ $\{1\} \cup D$ for $D$ a conjugacy class. For example consider the conjugacy class of a 3-cycle in the symmetric group of degree 3, this satisfies $K K^{-1}=\{1\} \cup K$. More examples are given in [5] and they prove the following result.

Theorem 3. [5, Theorem C] Let $K$ be a conjugacy class of a finite group $G$ and suppose $K K^{-1}=\{1\} \cup D$ where $D$ is a conjugacy class of $G$. Then $|D|$ divides $|K|(|K|-1)$ and $\langle K\rangle /\langle D\rangle$ is cyclic. In addition,

(1) If $|D|=|K|-1$ then $\langle K\rangle$ is metabelian. More precisely, $\langle D\rangle$ is $p$ elementary abelian for some prime $p$. 
(2) If $|D|=|K|$ then $\langle K\rangle$ is soluble with derived length at most 3.

(3) If $|D|=|K|(|K|-1)$ then $\langle K\rangle$ is abelian.

We apply the following combinatorial result, which appears in [8], to show that the situation in (1) is more common than the above implies.

Lemma 4. [8] Let $X$ be a finite subset of a nonabelian group $G$. If $\left|X^{-1} X\right|<$ $\frac{3}{2}|X|$ then $X X^{-1}$ and $X^{-1} X$ are both finite groups which are conjugate to each other. In particular, $X$ is contained in the right-coset (or left-coset) of a group of order less than $\frac{3}{2}|X|$.

Proof of Theorem C. $K$ is the conjugacy class $x^{G}$ and the hypotheses ensure that $\left|K K^{-1}\right|<\frac{3}{2}|K|$. So, by the previous lemma, $K K^{-1}$ is a finite group. Thus either $\langle D\rangle=\{1\} \cup D$ or $\langle D\rangle=\{1\} \cup D \cup D^{-1}$ respectively. It follows that $\langle D\rangle$ is $p$-elementary abelian (as it is a minimal normal subgroup). Further, $\langle K\rangle=\langle x\rangle[x, G]=\langle x\rangle\langle D\rangle$ and thus $K$ is metabelian.

Acknowledgements. The author would like to thank the Isaac Newton Trust for supporting her sabbatical during which time this research took place.

\section{References}

[1] A. Arad and M. Herzog, Products of conjugacy classes in groups, Lecture Notes in Mathematics, vol. 1112, Springer-Verlag, Berlin, 1985.

[2] E. Breuillard, A brief introduction to approximate groups, MSRI publication, Vol 61, 2013.

[3] A. Beltrán, R. Camina, M.J. Felipe and C. Melchor, Powers of conjugacy classes in a finite group, to appear in Annali di Matematica DOI: 10.1007/s10231-019-008852-2.

[4] A. Beltrán and M.J. Felipe, Cosets of normal subgroups and powers of conjugacy classes, preprint.

[5] A. Beltrán, M.J. Felipe and C. Melchor, Multiplying a conjugacy class by its inverse in a finite group, Israel J. Math 227 (2018), no. 2, 811-825. 
[6] G.A. Freiman, On finite subsets of nonabelian groups with small doubling, Proc. Amer. Math. Soc. 140 (2012), no. 9, 2997-3002.

[7] R. Guralnick and G. Navarro, Squaring a conjugacy class and cosets of normal subgroups, Proc. Amer. Math. Soc. 144 (2016), no. 5, 1939-1945.

[8] T. Tao, An elementary non-commutative Freiman theorem, http://terrytao.wordpresss.com/2009/11/10/ an-elementary-noncommutative-freiman-theorem. 